\title{
Clinicopathological features of pulmonary artery and vein intimal sarcomas: case series of rare pulmonary vessel intimal sarcoma
}

\author{
Hong-Qun Wang ${ }^{1,2 \#}$, Ai-Qun Sun ${ }^{2,3 \#}$, Peng $\mathrm{Liu}^{2}$, Wei Chen ${ }^{2}$, Chen $\mathrm{Cao}^{2}$, Xin Song ${ }^{2}$, Zhi-Gang Song \\ ${ }^{1}$ Department of Pathology, the Third People's Hospital of Bengbu City, Bengbu, China; ${ }^{2}$ Department of Pathology, the First Medical Center of the \\ Chinese People's Liberation Army (PLA) General Hospital, Beijing, China; ${ }^{3}$ Gaoqing County People's Hospital, Zibo, China \\ Contributions: (I) Conception and design: HQ Wang; (II) Administrative support: ZG Song, X Song; (III) Provision of study materials or patients: HQ \\ Wang, X Song, ZG Song; (IV) Collection and assembly of data: HQ Wang, AQ Sun, P Liu, W Chen, C Cao; (V) Data analysis and interpretation: \\ HQ Wang, AQ Sun; (VI) Manuscript writing: All authors; (VII) Final approval of manuscript: All authors. \\ \#These authors contributed equally to this work. \\ Correspondence to: Xin Song, Zhi-Gang Song. Department of Pathology, the First Medical Center of the Chinese People's Liberation Army (PLA) \\ General Hospital, Beijing, China. Email: sxin66@hotmail.com,13810410643@139.com.
}

\begin{abstract}
Background: Pulmonary vessel intimal sarcoma (IS) is rare.
Methods: We studied gross pathology, microscopic images and immunohistochemistry of 2 pulmonary artery ISs (PAISs) and 2 pulmonary vein ISs (PVISs), followed up the prognosis. The clinical manifestations of IS, imaging examination, electrocardiographic examination and serological examination were also studied.

Results: Grossly, the tumors were grayish yellow or grayish-white accompanied by local bleeding, and were manifested as sticky and slippery nodules. PAISs were located in the lumen of the pulmonary trunk or right ventricular outflow tract, and 1 of them had pedicle. PVISs were mainly located in the left atrium. Microscopically, the heteromorphic spindle cells were arranged in lobules and bundles, partially epithelioid, with visible mitotic figures. There were interstitial mucoid degeneration and local hemorrhage and necrosis/ infarction. Immunohistochemistry showed that vimentin, h-caldesmon and MDM2 were all positive, SATB2 (+, 3/4); Ki67 proliferation rate was 30-60\%; smooth muscle actin, desmin and CD56 were partially positive; cytokeratin and CD34 were locally positive; CD31, FLI-1, and ERG vascular markers were negative; and S-100 was negative. Case 4 showed MDM2 (12q15) amplification. Tumor markers were negative in venous blood; and lactate dehydrogenase increased in 2 cases. 3 patients died after surgery, 1 still survives after 14 months with lung and chest metastases for immunotherapy and 9 courses of chemotherapy.

Conclusions: IS is rare. Microscopically, it is mainly composed of spindle cells (or local epithelioid), along with interstitial mucoid degeneration. IS can differentiate into tumor of fibroblasts, bone, cartilage and smooth muscle, etc. The prognosis is poor.
\end{abstract}

Keywords: Pulmonary artery intimal sarcoma (PAIS); pulmonary vein intimal sarcoma (PVIS); clinicopathological features; prognosis; case series

Submitted Dec 19, 2020. Accepted for publication Jun 16, 2021.

doi: $10.21037 /$ tcr-20-3468

View this article at: https://dx.doi.org/10.21037/tcr-20-3468

\section{Introduction}

Intimal sarcoma (IS) is an extremely rare and highly malignant tumor arising in the tunica intima of large vessels of the systemic and pulmonary circulation. Pulmonary artery intimal sarcoma (PAIS) was first described in an autopsy by Mandelstamm in 1923 (1). PAIS often occurs in the main pulmonary trunk, with a polypoid growth pattern in the lumen, often invading the vascular wall 
or distant metastasis. Pulmonary vein intimal sarcoma (PVIS) often occurs in the left atrium and pulmonary vein. Clinically, PVIS is even rarer than PAIS (2). The most common clinical manifestation of pulmonary endovascular sarcoma is shortness of breath, followed by chest pain, cough, hemoptysis and syncope, etc. Due to its nonspecific clinical manifestations, pulmonary vessel IS is often misdiagnosed as cardiovascular disease, pulmonary embolism, or myxoma. Therefore, a better understanding of pulmonary vessel IS is required. Here, we explore the clinicopathological features of IS, combined with literature, to improve our understanding of the disease and facilitate early diagnosis and treatment. We present the following article in accordance with the AME Case Series reporting checklist (available at https://dx.doi. org/10.21037/tcr-20-3468).

\section{Methods}

\section{Patient data}

We collected 4 cases of IS in the Department of Pathology, the First Medical Center of PLA General Hospital from January 2018 to December 2020. As of March 26, 2021, each patient was followed up by telephone. The age of the patients ranged from 24 to 68 years (median: 59.5 years; mean: 52.8 years). There were 2 men and 2 women. All 4 patients underwent tumor resection. Case 3 also underwent right lower lung resection. Case 2 has been receiving 9 courses of chemotherapy 5 months after operation.

The clinicopathological data were analyzed, including gross pathological examination, hematoxylin-eosin (HE) staining, immunohistochemistry and fluorescence in situ hybridization (FISH). We also investigated clinical manifestations, imaging examination, electrocardiography (ECG), tumor markers and biomarkers of venous blood.

\section{Specimen preparation}

The postoperative specimens were fixed in $4 \%$ neutralbuffered formalin prior to routine dehydration and embedding. The blocks were then cut into sections, stained with $\mathrm{HE}$, and observed under light microscopy.

\section{Immunobistochemistry and FISH}

Antibodies and universal one-step kits were purchased from ZSQB-Bio (Beijing, China). All the antibodies were prepared at working concentrations, and all the tests were performed according to EnVision method. 3,3'-Diaminobenzidine dye was used for staining. The FISH kit was from Anbiping Pharmaceutical Technology Co. Ltd., and operated according to the instructions.

The study was conducted in accordance with the Declaration of Helsinki (as revised in 2013). The study was approved by institutional ethics committee of the first medical center of the Chinese PLA general hospital. Individual consent for this retrospective analysis was waived.

\section{Results}

\section{Clinical manifestations, auxiliary examination results, treatment and follow-up}

\section{Case 1}

Case 1 was a 67-year-old man with PAIS. In September 2018, he experienced heart palpitations and discomfort after physical activity but did not see a doctor. In October 2019, these symptoms worsened when he had mild activity and he visited a doctor. He had a history of smoking and alcohol consumption.

Venous blood tests showed increased levels of lactate dehydrogenase (LDH) (455.2 U/L) and $\gamma$-glutamyl transferase $(121.9 \mathrm{U} / \mathrm{L})$ but tumor markers were not measured. An ST-T abnormality was observed on ECG. The results of imaging examinations are shown in Table 1 (Figure 1).

The mass was resected. The mass in the pulmonary artery was lumpy meat-like, occupying the main and left pulmonary artery, and the right pulmonary artery was also invaded. The mass attached to the ventricular septum. However, the mass was widespread and no obvious pedicle was seen.

Repeated computed tomography (CT) 11 days after surgery showed that there was no obvious abnormal echo in the lumen. The patient had good postoperative recovery and was discharged after incision healing. However, 6 months later, the patient died of extensive metastasis of both lungs.

\section{Case 2}

Case 2 was a 24-year-old man with PAIS. He suffered from cough and fever for 2 weeks, along with chest pain. $\mathrm{He}$ had smoked occasionally for 7 years. His cardiac rhythm was regular, with grade III/6 systolic blowing murmurs at the right sternal border in the $3 \mathrm{rd}$ and 4 th intercostal 
Table 1 Imaging findings of the 4 cases

\begin{tabular}{|c|c|c|c|}
\hline Case & CT & Ultrasound & Magnetic resonance imaging \\
\hline 2 & $\begin{array}{l}\text { A lobulated mass with a long T2 signal, } \\
\text { partially enhancing with contrast- } \\
\text { enhanced scan. Multiple nodules } \\
\text { around both lungs. Embolizing left } \\
\text { lower pulmonary artery }\end{array}$ & $\begin{array}{l}\text { A space-occupying lesion in the right } \\
\text { ventricular outflow tract }\end{array}$ & Not performed \\
\hline 4 & Not performed & $\begin{array}{l}\text { A solid, slightly hyperechoic mass in the } \\
\text { left atrium, attached to the middle of the } \\
\text { atrial septum. Myxoma was considered. }\end{array}$ & Not performed \\
\hline
\end{tabular}

PET-CT, positron emission tomography-computed tomography; DWI, diffusion-weighted imaging.

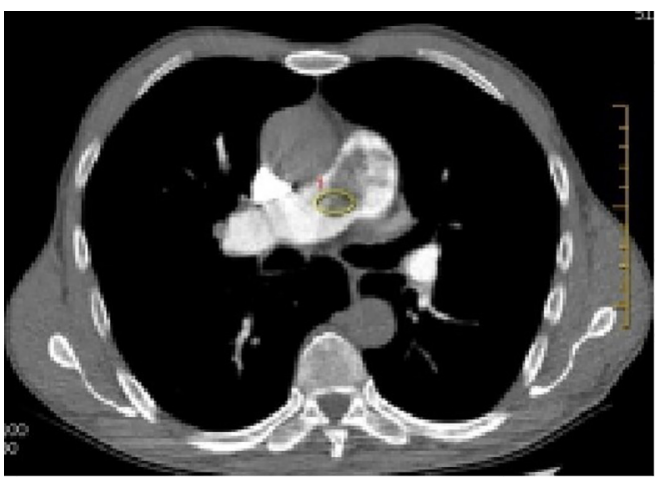

Figure 1 CT of Case 1 revealed a space-occupying lesion in the pulmonary artery.

spaces. ECG showed left ventricular high voltage. Venous blood tests showed normal tumor markers, whereas serum pepsinogen I level was low $(60.10 \mathrm{ng} / \mathrm{mL})$. The imaging findings are shown in Table 1.
The mass in the right ventricular outflow tract was removed. The tumor had a gray-white surface and fragile texture. It connected with the anterior wall of the right outflow tract through its pedicle. Part of the tumor also invaded the pulmonary artery valve, which was also removed.

Five months after surgery, the patient was admitted to hospital for immunotherapy and 9 courses of chemotherapy for lung and chest metastases. The patient still survives after 14 months.

\section{Case 3}

Case 3 was a 52-year-old woman with PVIS. She visited a local hospital because of chest pain and cough in August 2018. She had a history of smoking and alcohol consumption for $>20$ years. The preoperative diagnosis was space-occupying lesions in the right pulmonary vein and left atrium.

ECG showed T-wave abnormalities. Tumor markers 

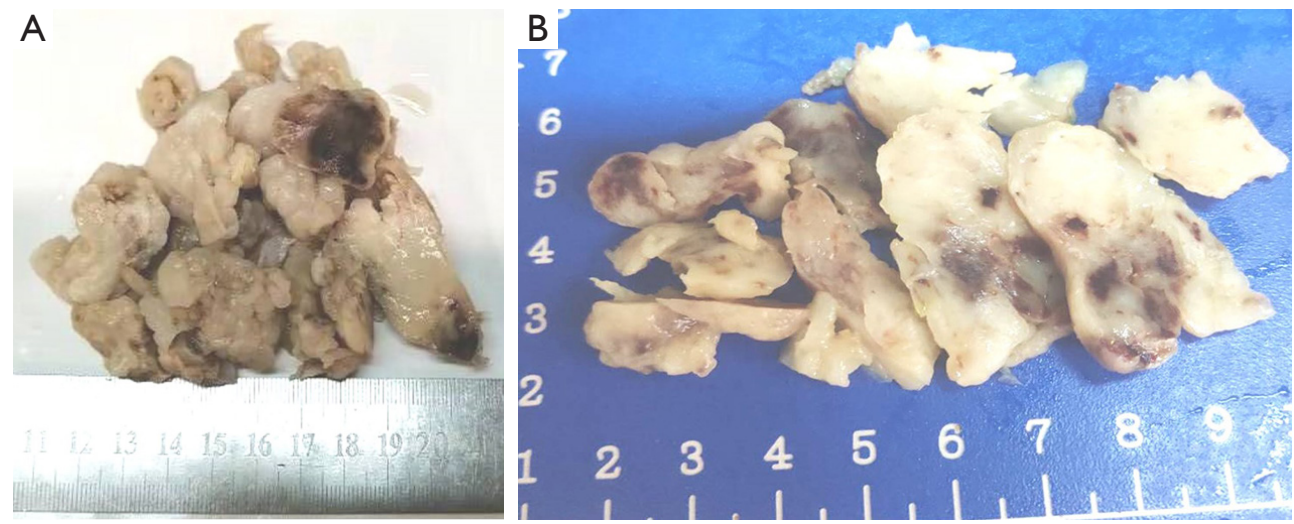

Figure 2 Gross pathological specimens. The tumors were yellowish in color and lobulated, with local bleeding. (A) Case 1 ; (B) Case 2.

were negative in venous blood, except for elevated serum ferritin $(152.10 \mathrm{ng} / \mathrm{mL})$. Blood biochemical tests showed increased low-density lipoprotein cholesterol $(4.51 \mathrm{mmol} / \mathrm{L})$, increased total cholesterol $(6.32 \mathrm{mmol} / \mathrm{L})$, and increased triglyceride $(2.11 \mathrm{mmol} / \mathrm{L})$. The imaging findings are shown in Table 1.

The tumor in the left atrium was tightly attached to the inferior wall of the left atrium and the interatrial septum and had an unclear boundary with the right inferior pulmonary vein. The tumors in the left atrium and pulmonary vein were resected, along with the right lower lung.

After surgery, the patient developed low cardiac output syndrome and received symptomatic treatments including cardiotonics, diuretics, antimicrobials, and myocardial nutrition. She left the intensive care unit (ICU) on postoperative day 5 . Unfortunately, the patient died from sudden cardiac arrest 14 days after surgery, which was believed to be due to acute pulmonary embolism.

\section{Case 4}

Case 4 was a 68 -year-old woman who presented with dizziness for $>2$ weeks and lasted for 2 minutes. She had chest pain and bilateral pleural effusion 2 months ago.

Serum examination showed 2 elevated markers, nonspecific enolase (NSE; $25.57 \mathrm{ng} / \mathrm{mL}$ ) and LDH (271.0 U/L).

The tumor was found to occupy the left atrium, no obvious pedicle, partial myxoid, tightly attached to right pulmonary vein and right wall of left atrium. The tumor was removed.

The patient died of relapse and lung metastasis $>2$ months after the operation.

\section{Pathological results: gross pathology, microscopic images, immunobistochemistry and diagnosis}

\section{Gross pathology}

Cases 1, 2 and 4 had clusters of light-yellow nodular or broken tissues of moderately hard consistency. The cut surface was light yellow, and there was a scattered gray-red area due to bleeding. The tumor was felt sticky and slippery (Figure 2). Case 3 had a cluster of gray-white tissues and moderately hard consistency in some areas. The cut surface was gray-white. Some tumor tissues were covered with vessel wall. Tumor size was as follows: $\sim 10.5 \mathrm{~cm} \times 9.2 \mathrm{~cm}$ $\times 2.1 \mathrm{~cm}$ for Case $1 ; 7.5 \mathrm{~cm} \times 5.5 \mathrm{~cm} \times 4.0 \mathrm{~cm}$ for Case 2, $\sim 11.5 \mathrm{~cm} \times 8.0 \mathrm{~cm} \times 2.0 \mathrm{~cm}$ for Case $4 ; \sim 9.5 \mathrm{~cm} \times 6.5 \mathrm{~cm} \times$ $2 \mathrm{~cm}$ for Case 3 and tumor size $3.0 \mathrm{~cm} \times 2.0 \mathrm{~cm} \times 1.8 \mathrm{~cm}$ in right lower lung.

\section{Light microscopy}

Spindle-shaped tumor cells, some may be epithelioid. Stromal myxoid change was obvious, with lobules, bundles and sheet arrangement, and abundant small blood vessels (Figures 3-6). Mitotic figures were visible. The tumor cells had abundant cytoplasm, with partial mild eosinophilic staining. Small nucleoli were seen in 2 cases. Hemorrhage, infarction and necrosis were found in some areas in 3 cases. A small number of lymphocytes, histiocytes and neutrophils were infiltrated in 2 cases, and a small number of multinucleated giant cells were found locally. Case 4 and 2 had focal mucinous lacustrine area, and Case 2 had focal myocardial invasion. The tumor shape in Case 3 was somewhat different. It showed mild to moderate heteromorphism of cells, no nucleoli, obvious cartilage and 

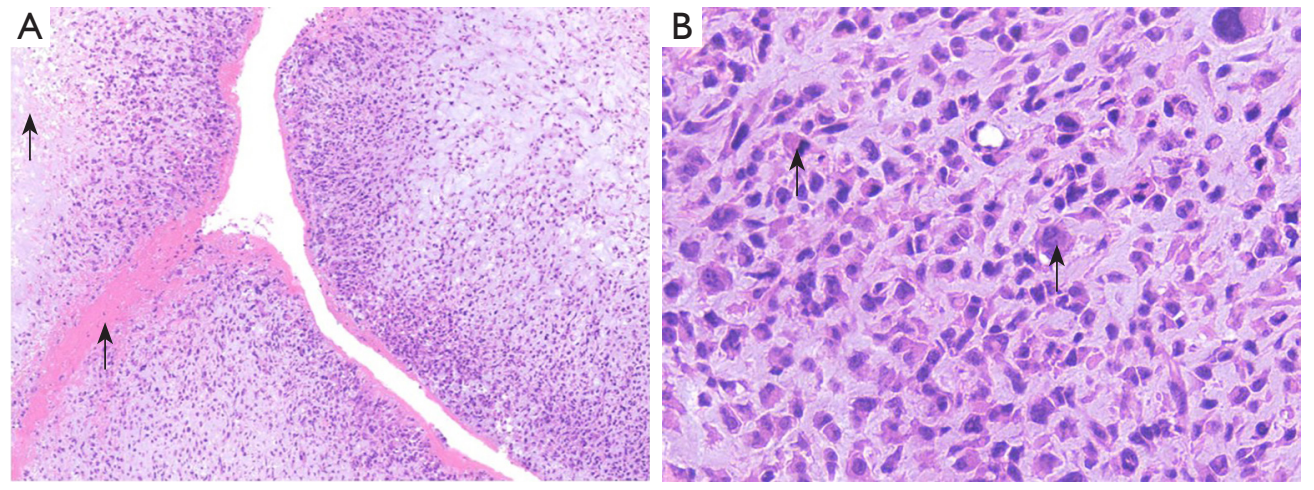

Figure 3 Case 1. (A) The tumor was lobulated, with myxoid degeneration, local hemorrhage and infarction (arrow), uneven cell density and dense perivascular cells $(\mathrm{HE} \times 100)$. (B) The tumor cells were epithelial-like, with obvious cell atypia and visible mitotic figures (arrow) $(\mathrm{HE} \times 400)$.
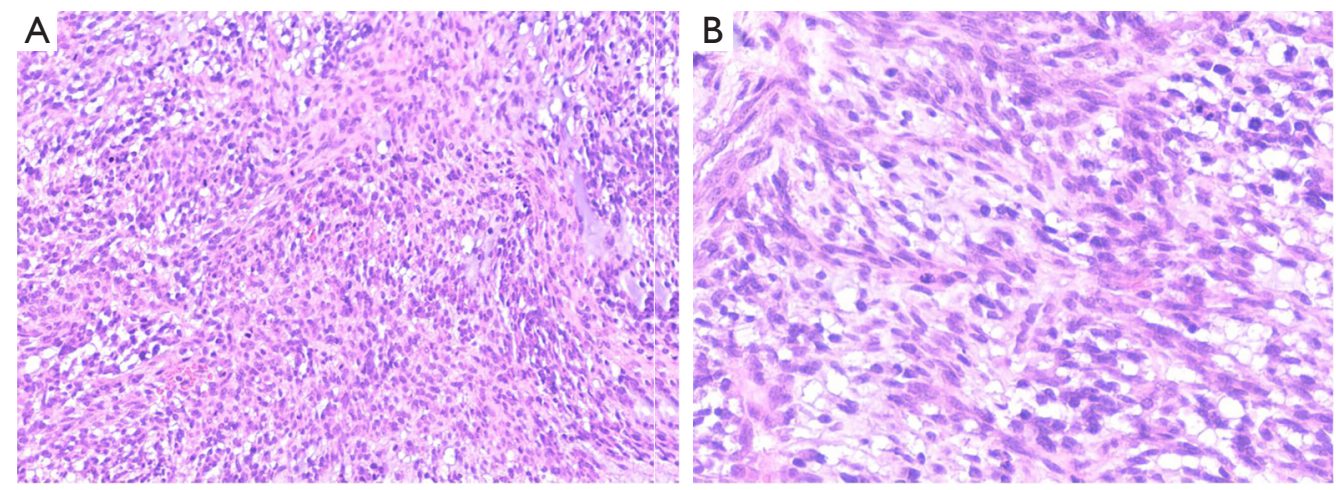

Figure 4 Case 2. (A) Tumor cells were arranged in bundles or sheets, uneven cell density, and mucoid degeneration was seen in some areas (HE $\times 100)$. (B) Tumor cells (partial epithelial cells) had obvious atypia, fusiform appearance, and nucleoli were not obvious (HE $\times 400)$.

bone tissue, invasion of lung tissue, no hemorrhage, no infarction and necrosis.

\section{Immunohistochemical staining (Table 2), FISH and pathological diagnosis \\ * $\quad$ Case 1 (Figure 7). PAIS.}

* $\quad$ Case 2 (Figure 8). PAIS. Immunohistochemistry also showed: CD99(+), P16(slightly +), Syn (weakly +); and CDK-4, calcitonin, WT-1, FLI-1, EGR and Dog-1 were negative.

* Case 3. EMA(-). PVIS with cartilage and bone differentiation.

- Case 4. PVIS. Immunohistochemistry also showed: Syn-, CD117-, CD99-. FISH showed MDM2 amplification (12q15).

\section{Discussion}

According to the International Agency for Research on Cancer (IARC) of the World Health Organization, IS is defined as a malignant tumor arising in the tunica intima of large blood vessels throughout the body, particularly in the pulmonary circulation. The tumor grows in the lumen and blocks local blood vessels, and tumor emboli spread to the surrounding organs. The prevalence of IS is extremely low $(\sim 0.001 \%)$. However, it has also been reported that IS occurs in large veins and the endocardium; the main part of PVIS is located in the left atrium, and part of it extends into the pulmonary vein $(2,3)$. ISs grow like polyps in the lumen of the blood vessel, and often spread distally along the recipient vessels, which in turn causes narrowing or even blockage of the blood vessels. Among the 4 cases presented 

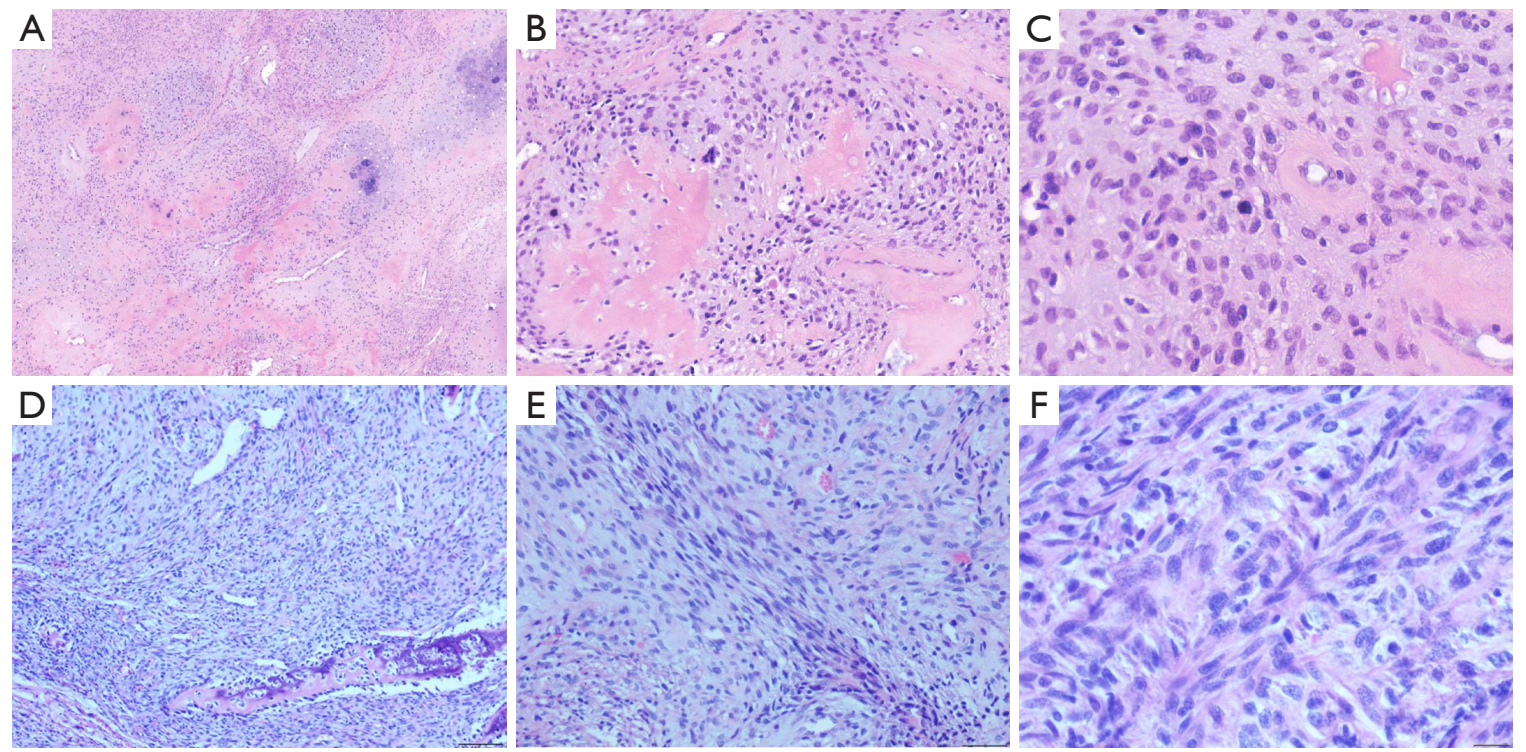

Figure 5 Case 3. (A-C) Tumor tissue was lobulated and differentiated into cartilage and bone. Cells were heteromorphic, nucleoli were not obvious, and mitotic figures were visible (A: HE $\times 50$; B: $\mathrm{HE} \times 200$; C: $\mathrm{HE} \times 400)$. (D-F) Fusiform cells were arranged in bundles and braid, along with interstitial mucoid changes. Cells were slightly heteromorphic and the nucleoli were not obvious $(\mathrm{D}$ : HE $\times 100$; E: HE $\times 200$; F: $\mathrm{HE} \times 400)$.
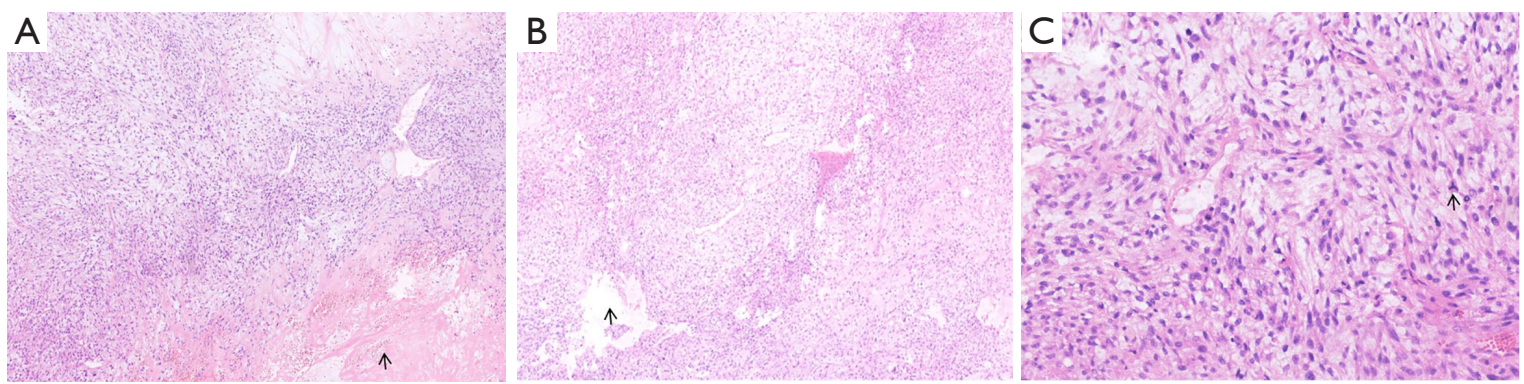

Figure 6 Case 4. (A) Tumor was lobulated, with myxoid degeneration, local hemorrhage and infarction (arrow), and uneven cell density (HE $\times 50)$. (B) Blood sinuses were abundant, and there were small mucus lakes (arrow) $(\mathrm{HE} \times 50)$. (C) Tumor cells were spindle type and mitotic figures were visible (arrow) $(\mathrm{HE} \times 200)$.

here, PAIS in Case 1 filled the main and left pulmonary artery and invaded the right pulmonary artery. PAIS in Case 2 was connected with the anterior wall of the right outflow tract through its pedicle, and part of it invaded the pulmonary artery valve. PVIS in Case 3 was located in the left atrium, tightly attached to the inferior wall of the left atrium and local interatrial septum, and invaded the right inferior pulmonary vein. During surgery in Case 4, it was found that the tumor occupied the left atrium, some of which was mucinous, without an obvious pedicle, and tightly attached to the right pulmonary vein and right wall of the left atrium.
IS can occur over a wide age range, with an average age of onset of 48.4 years (4). In the present series, median age was 59.5 years and mean age 52.8 years. There were 2 men with PAIS and 2 women with PVIS. The most common clinical symptom of IS is shortness of breath, followed by chest and back pain, cough, hemoptysis, weight loss, syncope, fever, and even sudden death. The general signs include systolic ejection murmurs, cyanosis, peripheral edema, jugular vein engorgement, hepatomegaly, and clubbing fingers. In our cases, the main clinical manifestations were chest pain and cough; patients experienced palpitation and shortness of breath, 1 had fever, and 1 had dizziness. 


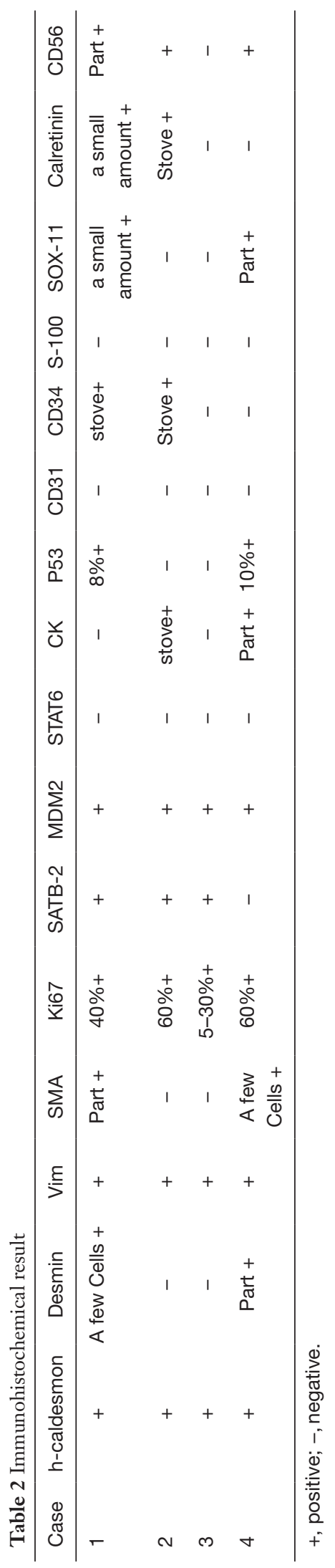

IS is often misdiagnosed as myxoma during preoperative imaging examinations. Myxoma is usually located in the left atrium, accounting for $\sim 50 \%$ of primary cardiac tumors. It is common in middle-aged women, and almost all of them are solitary, polypoid or lobulated, with pedicles (5). Under the microscope, the composition is mainly myxoid stroma, with scattered round, polygonal astrocytes, without heteromorphism (5). Imaging shows filling defects, uniform soft tissue density, and some irregular high-density calcification (6). In our series, the possibility of myxoma was considered in all 4 cases after auxiliary examinations. On CT, IS is often located in the pulmonary artery, with low-density filling defect and uneven density. Solid extension of the proximal pulmonary artery branch is highly suggestive of sarcoma, especially in the presence of pulmonary nodules, enlargement of the heart and decreased vascular distribution (7). The vessels in the lesion may be dilated, and the tumor often infiltrates the aortic valve and right pulmonary vein (7). CT revealed filling defects in our 3 patients, suggesting the presence of space-occupying lesions, which were partially enhanced after enhancement. The tumor could extend to the branches of blood vessels, and there were usually multiple metastases in both lungs. The pathological difference was mainly tumor cell heteromorphism of IS, the high proliferation index of Ki67 and amplification of MDM2.

Echocardiography showed space-occupying lesions in the hearts of our 4 patients. Echocardiography can also display the relationship between IS and artery wall. IS displays solid uneven echoes under color Doppler ultrasound, while thrombi show uniform weak echoes. Myxoma was suspected after ultrasonography in our cases, but echocardiography shows that myxoma can move back and forth with movement of the heart (8). In typical PAIS patients, chest $\mathrm{X}$-ray shows enlarged hilar shadow, nodular shadow inside the lungs, and enlarged heart shadow (9). However, chest $\mathrm{X}$-ray is of little value for atypical pulmonary IS. Magnetic resonance imaging (MRI) is also valuable for diagnosis of IS. In the present series, MRI in Case 2 showed slightly long $\mathrm{T} 1$ and long $\mathrm{T} 2$ roundish space-occupying signals in the right ventricular outflow tract and slightly high signal on diffusion-weighted imaging. These signals were not markedly enhanced in the early stage of enhancement but showed uneven continuous enhancement with time. Case 3 was considered to have low-grade malignancy.

There are other tumors that need to be differentiated. (I) Patients with cardiac and large vessel tumor emboli usually have a history and corresponding clinical symptoms 


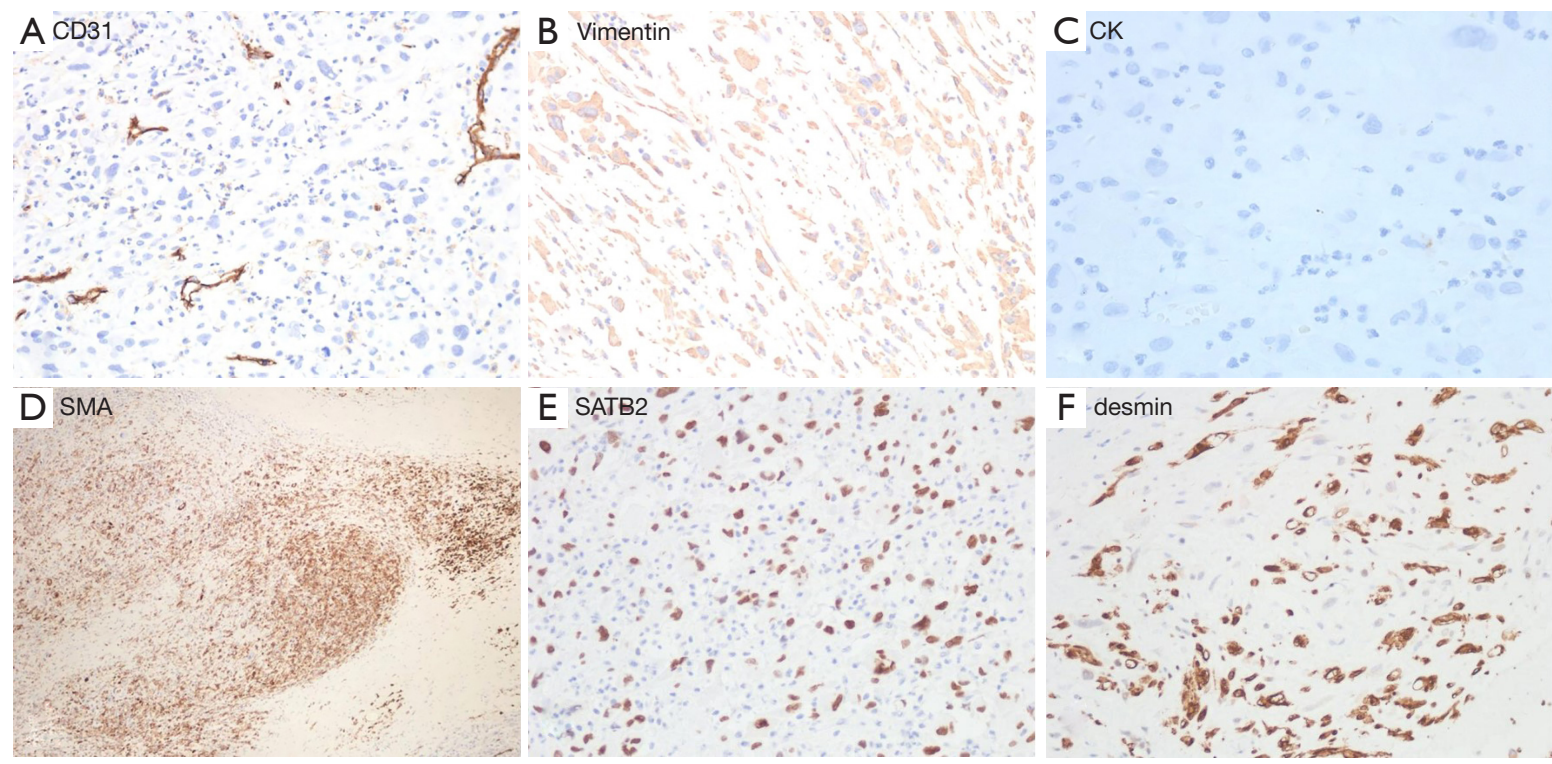

Figure 7 Immunohistochemical staining results of Case 1. (A) CD31, ×200; (B) vimentin, ×200; (C) CK, ×400; (D) SMA, ×40; (E) SATB2, $\times 200$; (F) desmin, $\times 200$.
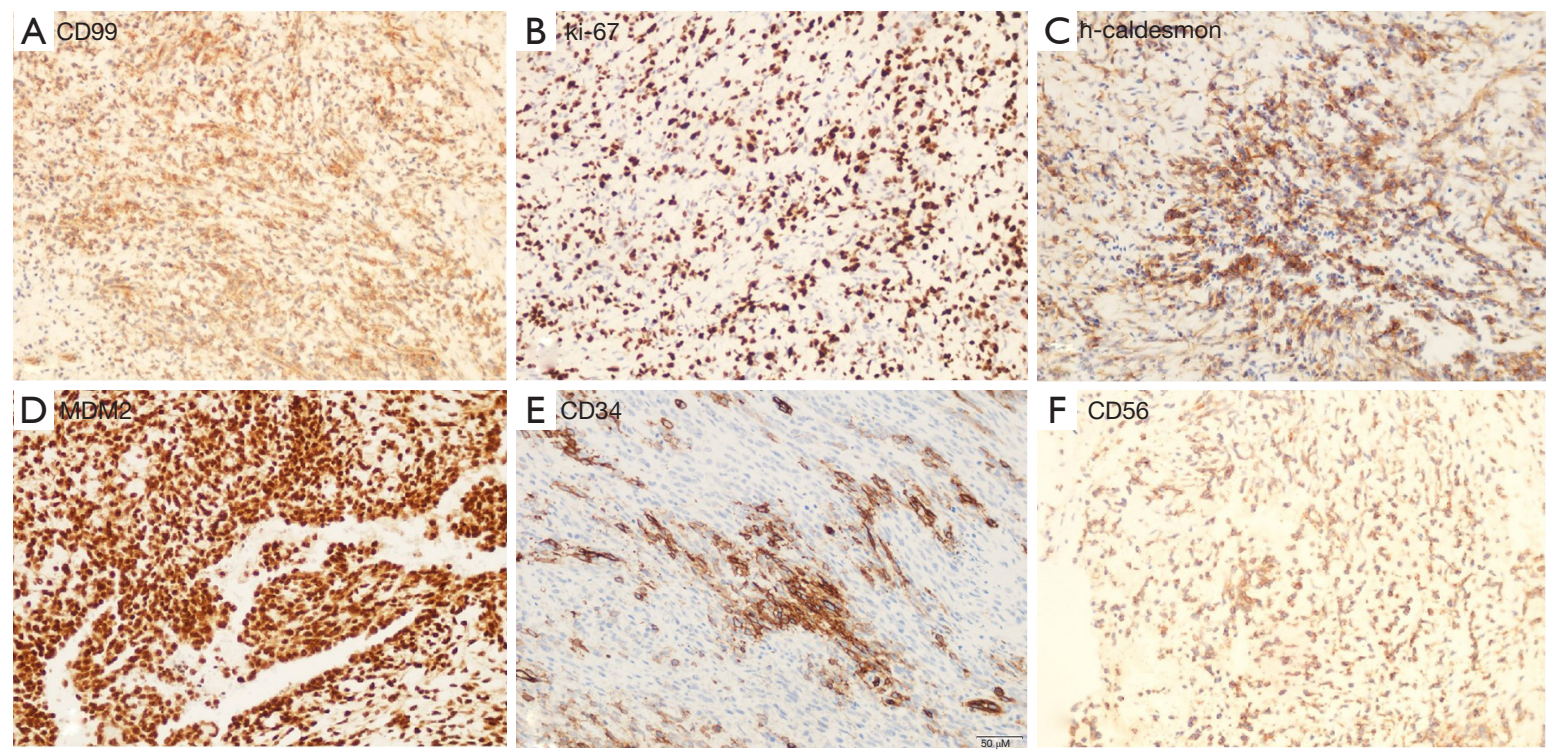

Figure 8 Immunohistochemical staining results of Case 2. (A) CD99, ×200; (B) Ki-67, ×200; (C) h-caldesmon, ×200; (D) MDM2, ×200; (E) CD34, ×200; (F) CD56, ×200.

of primary tumor, which are more common than primary tumor of the cardiovascular system, and most of them are metastatic cancer $(5,10)$. CT shows eccentric stenosis of the pulmonary artery, and the extent of involvement is limited (10). CT of IS shows that the pulmonary artery is filled with persistent soft tissue mass, and the external wall of the dilated vessels is uneven, indicating infiltration and dissemination of the tumor tissue (10). In this group of ISs, tumor thrombi were also found and all of them reached the lung first. Immunohistochemistry, morphology and medical history can help to differentiate diagnosis. (II) Leiomyosarcoma is rare in large blood vessels, and slightly 
more common in large veins. Generally, it is polypoid or nodular and adheres to the wall of blood vessels (11); it lacks myxoid stroma (except myxoid leiomyosarcoma); and the cytoplasm of tumor cells is eosinophilic. In this group of ISs, the general shape is similar, but IS has stick and slippery feeling, local hemorrhage and infarction, lobulated arrangement, and myxoid stroma. Smooth muscle markers such as SMA and desmin are diffusely positive in leiomyosarcoma, but they are not diffusely expressed in ISs. (III) Rhabdomyosarcoma is rare in the cardiovascular system, mostly occurs in adolescents and children, and usually consists of tumor cells with eosinophilic cytoplasm, nuclear deviation, and diverse cell morphology. Especially in embryonal rhabdomyosarcoma, there is myxoid degeneration in the stroma and dense tumor cells around the blood vessels, which is similar to IS. Age, morphology and immunohistochemistry are helpful for the differentiation. (IV) Angiosarcoma rarely originates from large blood vessels. The morphology and immunohistochemical staining of vascular endothelium are helpful in the differential diagnosis. IS also has focal CD34 positive. It is difficult to differentiate IS with imaging.

ECG abnormalities were found in 3 cases: ST-T abnormality in Case 1, left ventricular high voltage in Case 2 , and $\mathrm{T}$ abnormality in Case 3.

Venous blood tumor markers showed no obvious abnormalities in 3 cases and were not measured in the remaining patient. Only Case 4 had mild elevation of NSE. In 2 of the 3 cases, biochemical tests showed elevated LDH. It has been reported that most IS patients have elevated LDH levels (6/9) (12). D-dimer level is normal (12), but it is elevated in patients with thrombosis.

IS s often exhibit fibroblastic or myofibroblast differentiation. They may originate from endothelial cells, fibroblasts, and fibroblasts of the intima. Case 1 was considered to probably originate from the interventricular septum, Case 2 from the right ventricular outflow tract, and Cases 3 and 4 from the atrial septum. Microscopically, IS is mainly composed of spindle cells, along with scattered epithelioid cells. These cells have different degrees of atypia. Mitotic figures and interstitial mucoid degeneration are visible. There are various differentiations, including differentiations of osteosarcoma, chondroid, leiomyosarcoma, angiosarcoma, and rhabdomyosarcoma $(9,13-16)$. In our series, obvious cartilage and bone tissues were clearly observed under microscopy in a PVIS patient, showing that the tumor was differentiating into osteosarcoma. Infarction and necrosis were seen in some areas, along with scattered bleeding, and the removed tumors were felt sticky and slippery in 3 patients. Microscopically, all the tumors were lobulated; the interstitial mucoid degeneration was obvious, and there were abundant small blood vessels. The tumor cells were mainly spindle cells (partially epithelial cells), and they were arranged in bundles or sheets. The cytoplasm of the cells was abundant, along with unclear nucleoli and poorly demarcated boundaries. Mitotic figures were visible in all 4 cases. A small number of multinuclear tumor cells was found in 3 cases.

In the present study, immunohistochemistry showed positive vimentin, h-caldesmon, and MDM2; Ki67 proliferation rate was $30-60 \%$; SMA and desmin were partially positive; cytokeratin (CK) was focally positive in 2 cases; SATB2 was positive in 3 cases; CD31, FLI1 and ERG vascular markers were negative in tumor cells and positive in blood vessels in 1 case; S-100, ER, PR and EMA were negative; and CD99 was positive in $1 / 2$. These findings were consistent with previous results $(2,13-18)$, which showed that vimentin was diffusely positive, SMA and desmin were positive or locally positive, CK was positive in a small number of cases, and osteopontin was positive. The Ki-67 proliferation rate was $5-80 \%$, and CD117, CD68, p53 and bcl 2 were focally positive (8 cases). The literature also showed S-100, CD31, CD34, factor VIII, ERG, D2-40, SOX-10, ER and PR were negative. Immunohistochemistry showed high expression of MDM2, with amplified MDM2 gene as one of its features. Most tumors showed consistent genetic changes (acquisition and amplification of the 12q1314 region) (13-18). EGFR may be positive (2). Li et al. (2) and Fan et al. (13) reported that CD34 was focally positive in ISs, which was the same in 2 of our patients. Also, Li et al. (2) found that CD99 and CD56 were focally positive, which was similar to our results: CD56 was partially positive in 3 of 4 cases and Syn was weakly positive in Case 2, suggesting the presence of neuroendocrine differentiation. The amplification of $S A S / C D K 4, M D M 2$, GLI and PDGFRA genes is related to the occurrence of PAIS (19). In our study, Case 4 also showed MDM2(12q15) amplification.

PAIS has a poor prognosis (4). Bode-Lesniewska et al. reported 8 PAIS patients and 7 of them died: one immediately after surgery and the remaining 6 within 8-35 months (14). Similarly, Sebenik et al. (20) reported 14 patients and 11 of them died. Because most ISs are located inside the vascular lumen, surgical resection is the only treatment for short-term remission; however, surgery 
is high risk as it involves vital organs such as the heart and lungs. Miyai et al. (15) reported that 1 patient died of uncontrollable heart failure 2 days after surgery. In our series, 1 patient died from sudden cardiac arrest due to acute pulmonary embolism 14 days after surgery; 1 died of extensive metastasis of both lungs after 6 months; 1 survives for 14 months with lung metastasis, and was hospitalized for immunotherapy and chemotherapy 5 months after surgery; and the other died of recrudescence and lung metastasis $>2$ months after surgery. After surgery, multidisciplinary treatment including radiotherapy and chemotherapy may be administered. Xu et al. (21) reported a patient who had relapsed after surgery and achieved remission after chemotherapy. The disease was stable in the subsequent 19 months, and the main drug used was vinorelbine. Aso et al. (22) also described a case of unresectable PAIS; the patient was successfully treated using concurrent chemoradiotherapy with weekly paclitaxel, as well as subsequent chemotherapy.

\section{Conclusions}

Patients usually have chest pain, cough, palpitations, dizziness and other manifestations. The prognosis is poor. Early imaging is necessary for early detection and treatment. ECG abnormality and increased LDH also have indirect significance in the diagnosis of patients. Grossly, the tumors were grayish yellow or grayish-white accompanied by local bleeding, and were manifested as sticky and slippery nodules. Pulmonary vessel IS is mainly composed of spindle cells (or local epithelioid), along with interstitial mucoid degeneration. IS can be differentiated into tumors of fibroblasts, bone, cartilage, and smooth muscle, etc. Immunohistochemistry showed positive vimentin, h-caldesmon, and MDM2; SMA, desmin and CD56 were partially positive; CK and CD34 were locally positive; CD31, FLI-1 and ERG vascular markers were negative in tumor cells.

Due to the limited number of cases, more cases need to be studied in the future to understand the clinicopathological features of IS in detail.

\section{Acknowledgments}

We thank MedE Editing Group for the translation and English language editing. We also thank the teachers of molecular pathology department for FISH.

Funding: None.

\section{Footnote}

Reporting Checklist: The authors have completed the AME Case Series reporting checklist. Available at https://dx.doi. org/10.21037/tcr-20-3468

Data Sharing Statement: Available at https://dx.doi. org/10.21037/tcr-20-3468

Conflicts of Interest: All authors have completed the ICMJE uniform disclosure form (available at https://dx.doi. org/10.21037/tcr-20-3468). The authors have no conflicts of interest to declare.

Ethical Statement: The authors are accountable for all aspects of the work in ensuring that questions related to the accuracy or integrity of any part of the work are appropriately investigated and resolved. The study was conducted in accordance with the Declaration of Helsinki (as revised in 2013). The study was approved by institutional ethics committee of the first medical center of the Chinese PLA general hospital. Individual consent for this retrospective analysis was waived.

Open Access Statement: This is an Open Access article distributed in accordance with the Creative Commons Attribution-NonCommercial-NoDerivs 4.0 International License (CC BY-NC-ND 4.0), which permits the noncommercial replication and distribution of the article with the strict proviso that no changes or edits are made and the original work is properly cited (including links to both the formal publication through the relevant DOI and the license). See: https://creativecommons.org/licenses/by-nc-nd/4.0/.

\section{References}

1. Mandelstamm M. Über primäre Neubildungen des Herzens. Virchows Arch Pathol Anat 1923;245:43-5.

2. Li M, Liu YH, Zhuang HG, et al. Intimal sarcoma in left atrium and pulmonary vein: report of one case and literature review. Lin Chuang Yu Shi Yan Bing Li Xue Za Zhi 2009;25:535-37.

3. Zhang H, Macdonald WD, Erickson-Johnson M, et al. Cytogenetic and molecular cytogenetic findings of intimal sarcoma. Cancer Genet Cytogenet 2007;179:146-9.

4. Huo L, Moran CA, Fuller GN, et al. Pulmonary artery sarcoma: a clinicopathologic and immunohistochemical study of 12 cases. Am J Clin Pathol 2006;125:419-24. 
5. Rosai J. Rosai and Ackerman's surgical pathology. 10th ed. Chief translator: Zheng Jie. Peking University Medical Press: 2278-83.

6. Du P, Liu JY, Huang XY. Left atrial myxoma combined with coronary artery disease examined by 320 slice computed tomography. Yixue Ying Xiang Xue Za Zhi 2011;21:198-200.

7. Yi CA, Lee KS, Choe YH, et al. Computed tomography in pulmonary artery sarcoma: distinguishing features from pulmonary embolic disease. J Comput Assist Tomogr 2004;28:34-9.

8. You JX, Chen HM, Zhang WQ, et al. The imaging analysis of primary cardiac tumors. Yixue Zhen Duan Yu Jie Ru Fang She Xue 2008;17:253-56.

9. Bleisch VR, Kraus FT. Polypoid sarcoma of the pulmonary trunk: analysis of the literature and report of a case with leptomeric organelles and ultrastructural features of rhabdomyosarcoma. Cancer 1980;46:314-24.

10. Yue JQ, Guo XF, Zhang YGL, et al. An Analysis of the Diagnosis and Treatment of 17 Cases of Domestic Primary Artery Intimal Sarcoma. Hubei Ming Zu Xue Yuan Xue Bao (Medical Edition) 2012;29:16-21.

11. Wang J, Zhu XZ. Pathology of soft tissue tumors. 2nd ed, People's Health Publishing Press: 596.

12. Guo W, Zhang W, Huang X, et al. Clinical characteristics of 9 patients with pulmonary artery sarcoma. Zhonghua Xin Xue Guan Bing Za Zhi 2014;42:38-42.

13. Fan XS, Wang YH, Yue MZ, et al. Primary pulmonary artery intimal sarcoma: a case report and review of literature. Zhen Duan Bing Li Xue Za Zhi 2007;2:105-9.

14. Bode-Lesniewska B, Zhao J, Speel EJ, et al. Gains of 12q13-14 and overexpression of mdm2 are frequent findings in intimal sarcomas of the pulmonary artery.

Cite this article as: Wang HQ, Sun AQ, Liu P, Chen W, Cao C, Song X, Song ZG. Clinicopathological features of pulmonary artery and vein intimal sarcomas: case series of rare pulmonary vessel intimal sarcoma. Transl Cancer Res 2021;10(6): 3033-3043. doi: 10.21037/tcr-20-3468
Virchows Arch 2001;438:57-65.

15. Miyai K, Takeo H, Matsukuma S. Intimal sarcoma of the pulmonary trunk showing broad intimal extension and focal chondrosarcomatous differentiation: an autopsy case. Cardiovasc Pathol 2017;31:5-8.

16. Harbhajanka A, Dahoud W, Michael CW, et al. Cytohistological correlation, immunohistochemistry and Murine Double Minute Clone 2 amplification of pulmonary artery intimal sarcoma: A case report with review of literature. Diagn Cytopathol 2019;47:494-7.

17. Sejben A, Tiszlavicz L, Furák J, et al. Intimal sarcoma of pulmonary arteries. Orv Hetil 2020;161:232-6.

18. Lu P, Yin BB. Misdiagnosis of primary intimal sarcoma of the pulmonary artery as chronic pulmonary embolism: A case report. World J Clin Cases 2020;8:986-94.

19. Zhao J, Roth J, Bode-Lesniewska B, et al. Combined comparative genomic hybridization and genomic microarray for detection of gene amplifications in pulmonary artery intimal sarcomas and adrenocortical tumors. Genes Chromosomes Cancer 2002;34:48-57.

20. Sebenik M, Ricci A Jr, DiPasquale B, et al. Undifferentiated intimal sarcoma of large systemic blood vessels: report of 14 cases with immunohistochemical profile and review of the literature. Am J Surg Pathol 2005;29:1184-93.

21. Xu Y, Wang K, Geng Y, et al. A case of intimal sarcoma of the pulmonary artery successfully treated with chemotherapy. Int J Clin Oncol 2012;17:522-7.

22. Aso T, Terao M, Endo H, et al. A case of pulmonary artery intimal sarcoma successfully treated using concurrent chemoradiotherapy and subsequent chemotherapy. Int Cancer Conf J 2019;8:71-6. 